Proceedings of the Edinburgh Mathematical Society (2008) 51, 155-170 (C)

DOI:10.1017/S0013091505001860 Printed in the United Kingdom

\title{
A NEW CHARACTERIZATION FOR REGULAR BMO WITH NON-DOUBLING MEASURES
}

\author{
GUOEN HU ${ }^{1}$, XIN WANG ${ }^{1}$ AND DACHUN YANG ${ }^{2 *}$ \\ ${ }^{1}$ Department of Applied Mathematics, University of Information Engineering, \\ PO Box 1001-747, Zhengzhou 450002, People's Republic of China \\ (huguoen@eyou.com) \\ ${ }^{2}$ School of Mathematical Sciences, Beijing Normal University, Beijing 100875, \\ People's Republic of China (dcyang@bnu.edu.cn)
}

(Received 21 December 2005)

\begin{abstract}
Let $\mu$ be a positive Radon measure on $\mathbb{R}^{d}$ which satisfies $\mu(B(x, r)) \leqslant C r^{n}$ for any $x \in \mathbb{R}^{d}$ and $r>0$ and some fixed constants $C>0$ and $n \in(0, d]$. In this paper, a new characterization of the space $\operatorname{RBMO}(\mu)$, which was introduced by Tolsa, is given. As an application, it is proved that the $L^{p}(\mu)$-boundedness with $p \in(1, \infty)$ of Calderón-Zygmund operators is equivalent to various endpoint estimates.
\end{abstract}

Keywords: non-doubling measure; RBMO; $H^{1}$-atomic block; Calderón-Zygmund operator

2000 Mathematics subject classification: Primary 42B30; 42B20

\section{Introduction}

Let $\mu$ be a positive Radon measure on $\mathbb{R}^{d}$ which satisfies the following growth condition: there exist constants $C>0$ and $n \in(0, d]$ such that, for all $x \in \mathbb{R}^{d}$ and $r>0$,

$$
\mu(B(x, r)) \leqslant C r^{n},
$$

where $B(x, r)$ is the ball centred at some point $x \in \mathbb{R}^{d}$ and having radius $r$. The measure $\mu$ in (1.1) may not satisfy the doubling condition which is a key assumption in the analysis on spaces of homogeneous type. We recall that $\mu$ is said to satisfy the doubling condition if there exists some constant $C>0$ such that $\mu(B(x, 2 r)) \leqslant C \mu(B(x, r))$ for all $x \in \mathbb{R}^{d}$ and $r>0$. Some important examples of non-doubling measures as in (1.1) and the motivation for developing the analysis related to such measures can be found in $[\mathbf{1 3}]$. Note that the analysis with non-doubling measures plays an essential role in solving the long-standing open Painlevé problem posed by Tolsa in [12].

In recent years, there has been significant progress in the study of function spaces and boundedness of singular integral operators associated with non-doubling measures (see

\footnotetext{
* Author for correspondence.
} 
$[\mathbf{1}, \mathbf{2}, \mathbf{4}-\mathbf{7}, \mathbf{9}-\mathbf{1 3}])$. A prototypical work in this area is $[\mathbf{9}]$. In this remarkable work, Tolsa found a suitable substitute for the classical BMO space in this setting, $\operatorname{RBMO}(\mu)$, which is small enough to satisfy the properties of the classical BMO space and large enough that an $L^{2}(\mu)$ bounded Calderón-Zygmund operator is also bounded from $L^{\infty}(\mu)$ to $\operatorname{RBMO}(\mu)$. It has been proved that $\operatorname{RBMO}(\mu)$ plays a very important role in the study of function spaces and the boundedness of operators related to non-doubling measures $[\mathbf{1}, \mathbf{2}, \mathbf{9}]$. We should point out that BMO-type spaces with non-doubling measures were also considered by Mateu et al. in [4], and by Nazarov et al. in [6]. However, the BMO-type space in [4] cannot guarantee that an $L^{2}(\mu)$ bounded Calderón-Zygmund operator is bounded from $L^{\infty}(\mu)$ to this space (see [13]), and the BMO-type space in [6] does not satisfy the John-Nirenberg inequality.

The purpose of this paper is to establish a new characterization of $\operatorname{RBMO}(\mu)$. As an application of this, we prove that the $L^{p}(\mu)$-boundedness with $p \in(1, \infty)$ for a CalderónZygmund operator is equivalent to its several endpoint estimates, respectively. To state the main result, we first recall some definitions and notation.

By a cube $Q \subset \mathbb{R}^{d}$ we mean a closed cube whose sides are parallel to the axes and which is centred at some point of $\operatorname{supp} \mu$, and we denote its side length by $l(Q)$. Let $\alpha$ and $\beta_{d}$ be positive constants such that $\beta_{d}>\alpha^{n}$. For a cube $Q$, we say that $Q$ is $(\alpha, \beta)$ doubling if $\mu(\alpha Q) \leqslant \beta \mu(Q)$, where $\alpha Q$ denotes the cube concentric with $Q$ and having side length $\alpha l(Q)$. In what follows, if $\alpha$ and $\beta$ are not specified, by a doubling cube we mean a $\left(2,2^{d+1}\right)$-doubling cube. For two cubes $Q_{1} \subset Q_{2}$, set

$$
\delta_{Q_{1}, Q_{2}}=1+\sum_{k=1}^{N_{Q_{1}, Q_{2}}} \frac{\mu\left(2^{k} Q_{1}\right)}{\left[l\left(2^{k} Q_{1}\right)\right]^{n}},
$$

where $N_{Q_{1}, Q_{2}}$ is the least integer $k$ such that $l\left(2^{k} Q_{1}\right) \geqslant l\left(Q_{2}\right)$.

As usual, in what follows, we denote by $L_{\text {loc }}^{1}(\mu)$ the set of all locally integrable functions with respect to the measure $\mu$. We now recall the following definition of $\operatorname{RBMO}(\mu)$ in $[\mathbf{9}]$.

Definition 1.1. Let $\rho \in(1, \infty)$ be fixed. A function $f \in L_{\text {loc }}^{1}(\mu)$ is said to belong to the space $\operatorname{RBMO}(\mu)$ if there exists a constant $C_{1} \geqslant 0$ such that

$$
\sup _{Q} \frac{1}{\mu(\rho Q)} \int_{Q}\left|f(x)-m_{\tilde{Q}}(f)\right| \mathrm{d} \mu(x) \leqslant C_{1},
$$

and, for any two doubling cubes $Q_{1} \subset Q_{2}$,

$$
\left|m_{Q_{1}}(f)-m_{Q_{2}}(f)\right| \leqslant C_{1} \delta_{Q_{1}, Q_{2}},
$$

where the supremum is taken over all cubes centred at some point of $\operatorname{supp} \mu, \tilde{Q}$ is the smallest doubling cube of the form $2^{k} Q$ with non-negative integer $k$, and $m_{\tilde{Q}}(f)$ is the mean value of $f$ on $\tilde{Q}$, namely,

$$
m_{\tilde{Q}}(f)=\frac{1}{\mu(\tilde{Q})} \int_{\tilde{Q}} f(y) \mathrm{d} \mu(y) .
$$

The minimal constant $C_{1}$ in (1.3) and (1.4) is defined to be the $\operatorname{RBMO}(\mu)$ norm of $f$ and is denoted by $\|f\|_{\operatorname{RBMO}(\mu)}$. 
Remark 1.2. Tolsa [9] proved that the definition of $\operatorname{RBMO}(\mu)$ is independent of choices of $\rho, \alpha$ and $\beta_{d}$ provided that $\rho>1$ and $\beta_{d}>\alpha^{n}$. Also, we can obtain an equivalent definition for the space $\operatorname{RBMO}(\mu)$ by replacing cubes centred at points of $\operatorname{supp} \mu$ by all cubes in $\mathbb{R}^{d}$.

Definition 1.3. Let $\rho \in(1, \infty)$ and $p \in(0, \infty)$. A function $f \in L_{\text {loc }}^{1}(\mu)$ is said to belong to the space $\operatorname{RBMO}_{\rho}^{p}(\mu)$ if there exist a constant $C_{2} \geqslant 0$ and a collection of numbers $\left\{f_{Q}\right\}$ (i.e. for each cube $Q$, there exists a number $f_{Q}$ ) such that

$$
\sup _{Q} \frac{1}{\mu(\rho Q)} \int_{Q}\left|f(x)-f_{Q}\right|^{p} \mathrm{~d} \mu(x) \leqslant C_{2}^{p}
$$

and

$$
\left|f_{Q}-f_{R}\right| \leqslant C_{2} \delta_{Q, R}
$$

The minimal constant $C_{2}$ in (1.5) and (1.6) is defined to be the $\operatorname{RBMO}_{\rho}^{p}(\mu)$ norm of $f$ and is denoted by $\|f\|_{\mathrm{RBMO}_{\rho}^{p}(\mu)}$.

The main result in this paper is the following new characterization of $\operatorname{RBMO}(\mu)$.

Theorem 1.4. Let $\rho \in(1, \infty)$ and $p \in(0, \infty)$. The spaces $\operatorname{RBMO}_{\rho}^{p}(\mu)$ and $\operatorname{RBMO}(\mu)$ coincide and their norms are equivalent.

Note that if $\mu$ is the $d$-dimensional Lebesgue measure, then Theorem 1.4 is $[\mathbf{8}$, Corollary, p. 517]. Moreover, a version of Theorem 1.4 with respect to doubling measures was established by Long and Yang in [3].

Remark 1.5. The essential new part of Theorem 1.4 is the case $p \in(0,1)$. The case $p \in[1, \infty)$ of Theorem 1.4 is trivial by the Hölder inequality and the John-Nirenberg inequality for the space $\operatorname{RBMO}(\mu)$, which was established in [9].

To give an application of Theorem 1.4, we first recall the definition of the Hardy space with the measure satisfying (1.1), which was introduced by Tolsa in [9] (see also [11]).

Definition 1.6. Let $\rho \in(1, \infty)$. A function $b \in L_{\mathrm{loc}}^{1}(\mu)$ is said to be an $H^{1}(\mu)$-atomic block if

(i) there exists some cube $R$ such that $\operatorname{supp} b \subset R$,

(ii) $\int_{\mathbb{R}^{d}} b(x) \mathrm{d} \mu(x)=0$,

(iii) there are functions $a_{j}$ supported on cubes $Q_{j} \subset R$ and numbers $\lambda_{j} \in \mathbb{R}$ such that $b=\sum_{j=1}^{\infty} \lambda_{j} a_{j}$ and

$$
\left\|a_{j}\right\|_{L^{\infty}(\mu)} \leqslant\left(\mu\left(\rho Q_{j}\right) \delta_{Q_{j}, R}\right)^{-1}
$$

Set

$$
|b|_{H_{\mathrm{atb}}^{1, \infty}(\mu)}=\sum_{j=1}^{\infty}\left|\lambda_{j}\right| .
$$


For a function $f \in L^{1}(\mu)$, we say that $f \in H_{\mathrm{atb}}^{1, \infty}(\mu)$ if there are $H^{1}(\mu)$-atomic blocks $\left\{b_{j}\right\}_{j=1}^{\infty}$ such that

$$
f=\sum_{j=1}^{\infty} b_{j}
$$

with $\sum_{j=1}^{\infty}\left|b_{j}\right|_{H_{\mathrm{atb}}^{1, \infty}(\mu)}<\infty$. The $H_{\mathrm{atb}}^{1, \infty}(\mu)$ norm of $f$ is defined by

$$
\|f\|_{H_{\mathrm{atb}}^{1, \infty}(\mu)}=\inf \left\{\sum_{j=1}^{\infty}\left|b_{j}\right|_{H_{\mathrm{atb}}^{1, \infty}(\mu)}\right\},
$$

where the infimum is taken over all the possible decompositions of $f$ as above.

Remark 1.7. Tolsa [9, Proposition 4.1] states that the definition of $H_{\mathrm{atb}}^{1, \infty}(\mu)$ is independent of the choice of $\rho>1$.

Let $K$ be a locally integrable function on $\mathbb{R}^{d} \times \mathbb{R}^{d} \backslash\{x=y\}$ which has the property that, for all $x, y \in \mathbb{R}^{d}$ with $x \neq y$,

$$
|K(x, y)| \leqslant C|x-y|^{-n},
$$

and, for all $x, y, y^{\prime} \in \mathbb{R}^{d}$ with $|x-y| \geqslant 2\left|y-y^{\prime}\right|$ and $x \neq y$,

$$
\left|K(x, y)-K\left(x, y^{\prime}\right)\right|+\left|K(y, x)-K\left(y^{\prime}, x\right)\right| \leqslant C \frac{\left|y-y^{\prime}\right|^{\gamma}}{|x-y|^{n+\gamma}},
$$

where $\gamma \in(0,1]$ and $C>0$ are two constants independent of $x, y$ and $y^{\prime}$. Define the Calderón-Zygmund operator, $T$, formally by

$$
T f(x)=\int_{\mathbb{R}^{d}} K(x, y) f(y) \mathrm{d} \mu(y) .
$$

This integral may not be convergent for many functions. Thus, we consider the truncated operators $T_{\varepsilon}$ for $\varepsilon>0$ defined by

$$
T_{\varepsilon} f(x)=\int_{|x-y| \geqslant \varepsilon} K(x, y) f(y) \mathrm{d} \mu(y) .
$$

For $p \in(1, \infty)$, we say that the operator $T$ is bounded on $L^{p}(\mu)$ if $T_{\varepsilon}$ is bounded on $L^{p}(\mu)$ with its bound independent of $\varepsilon$. The boundedness of $T$ from $H_{\mathrm{atb}}^{1, \infty}(\mu)$ to $L^{1}(\mu)$, and from $L^{\infty}(\mu)$ to $\operatorname{RBMO}(\mu)$, is defined similarly. Also, we say that an operator $T$ is of restricted weak type $\left(H_{\mathrm{atb}}^{1, \infty}(\mu), L^{1}(\mu)\right)$ if there is a constant $C>0$ such that, for any $\varepsilon>0, \lambda>0$ and any $H^{1}(\mu)$-atomic block $b$,

$$
\mu\left(\left\{x \in \mathbb{R}^{d}:\left|T_{\varepsilon} b(x)\right|>\lambda\right\}\right) \leqslant C \lambda^{-1}|b|_{H_{\mathrm{atb}}^{1, \infty}(\mu)} .
$$

Tolsa [10] established the Calderón-Zygmund decomposition and proved that if $K$ satisfies (1.7) and (1.8), and $T$ is bounded on $L^{2}(\mu)$, then $T$ is also bounded from $L^{1}(\mu)$ to weak $L^{1}(\mu)$, and from $L^{p}(\mu)$ to itself for any $p \in(1, \infty)$. Using some results on $\operatorname{RBMO}(\mu)$ and $H_{\text {atb }}^{1, \infty}(\mu)$, Tolsa proved the following result (see [9, Theorem 8.1]). 
Theorem 1.8. For $\rho \in(1, \infty)$, let $K$ satisfy (1.7) and (1.8), and let $T$ be the corresponding Calderón-Zygmund operator. The following properties are equivalent:

(1) there is a constant $C>0$ such that, for any $\varepsilon>0$, cube $Q$ and bounded function $f$ with $\operatorname{supp} f \subset Q$,

$$
\frac{1}{\mu(\rho Q)} \int_{Q}\left|T_{\varepsilon} f(x)\right| \mathrm{d} \mu(x) \leqslant C\|f\|_{L^{\infty}(\mu)}
$$

(2) $T$ is bounded from $L^{\infty}(\mu)$ to $\operatorname{RBMO}(\mu)$;

(3) $T$ is bounded from $H_{\mathrm{atb}}^{1, \infty}(\mu)$ to $L^{1}(\mu)$;

(4) $T$ is bounded on $L^{p}(\mu)$ for any $p \in(1, \infty)$.

Invoking Theorem 1.4, we improve Theorem 1.8, giving the following result.

Theorem 1.9. Let $\rho \in(1, \infty)$, let $K$ satisfy (1.7) and (1.8), and let $T$ be the corresponding Calderón-Zygmund operator. The following properties are equivalent:

(i) $T$ is of restricted weak type $\left(H_{\mathrm{atb}}^{1, \infty}(\mu), L^{1}(\mu)\right)$;

(ii) for certain $\nu>0$, there is a constant $C>0$ such that, for any $\varepsilon>0, \lambda>0$, cube $Q$ and bounded function $f$ with $\operatorname{supp} f \subset Q$,

$$
\mu\left(\left\{x \in Q:\left|T_{\varepsilon} f(x)\right|>\lambda\right\}\right) \leqslant C \lambda^{-\nu} \mu(\rho Q)\|f\|_{L^{\infty}(\mu)}^{\nu} ;
$$

(iii) for certain $\sigma \in(0,1)$, there is a constant $C>0$ such that, for any $\varepsilon>0$ and any bounded function $f$ with $\operatorname{supp} f \subset Q$,

$$
\frac{1}{\mu(\rho Q)} \int_{Q}\left|T_{\varepsilon} f(x)\right|^{\sigma} \mathrm{d} \mu(x) \leqslant C\|f\|_{L^{\infty}(\mu)}^{\sigma} ;
$$

(iv) $T$ is bounded from $L^{\infty}(\mu)$ to $\operatorname{RBMO}(\mu)$;

(v) $T$ is bounded on $L^{p}(\mu)$ for any $p \in(1, \infty)$.

We remark that (ii) and (iii) of Theorem 1.9 are essentially equivalent. Obviously, (i) and (iii) of Theorem 1.9 are much weaker than (3) and (1) of Theorem 1.8, respectively. Moreover, the equivalence between (i) and (v) of Theorem 1.9 is not explicitly implied by Theorem 1.8. In the proof of Theorem 1.9, the essential part is that (iii) implies (iv), in which Theorem 1.4 plays a key role.

From Theorem 1.9, we can, furthermore, deduce the following result.

Corollary 1.10. Let $K$ satisfy (1.7) and (1.8), let $T$ be the corresponding CalderónZygmund operator and let $\Phi$ be a Young function such that, for any $t_{1}, t_{2} \geqslant 0$,

$$
\Phi\left(t_{1} t_{2}\right) \leqslant C \Phi\left(t_{1}\right) \Phi\left(t_{2}\right),
$$


and, for certain $\sigma \in(0,1)$,

$$
\int_{1}^{\infty} \Phi\left(\frac{1}{t}\right) t^{\sigma-1} \mathrm{~d} t<\infty
$$

If there is a constant $C>0$ such that, for any $\varepsilon>0, \lambda>0$ and bounded function $f$ with compact support,

$$
\mu\left(\left\{x \in \mathbb{R}^{d}:\left|T_{\varepsilon} f(x)\right|>\lambda\right\}\right) \leqslant C \int_{\mathbb{R}^{d}} \Phi\left(\frac{|f(x)|}{\lambda}\right) \mathrm{d} \mu(x),
$$

then $T$ is also bounded on $L^{p}(\mu)$ for any $p \in(1, \infty)$.

If we take $\Phi(t)=t \log ^{\gamma}(2+t)$ for $t \geqslant 0$ and certain $\gamma \geqslant 1$, Corollary 1.10 with this $\Phi$ then indicates that if $T$ is of weak type $\left(L \log ^{\gamma} L(\mu), L^{1}(\mu)\right)$, then $T$ is also bounded on $L^{p}(\mu)$ for $p \in(1, \infty)$. This cannot be deduced immediately from Theorem 1.8.

Throughout this paper, $C$ denotes a positive constant that is independent of the main parameters involved but whose value may differ from line to line. Constants with subscripts, such as $C_{1}$, retain their value at different occurrences. $f \sim g$ means that the ratio $f / g$ is upper bounded and lower bounded away from zero by constants that do not depend on the relevant variables in $f$ and $g . f \lesssim g$ is similar. For a $\mu$-measurable set $E$, $\chi_{E}$ denotes the characteristic function of $E$.

\section{Proof of Theorem 1.4}

We begin with some preliminary results.

Lemma 2.1. Let $p \in(0,1)$ and $1<\rho_{1}<\rho_{2}<\infty$. Then $\operatorname{RBMO}_{\rho_{1}}^{p}(\mu)$ and $\operatorname{RBMO}_{\rho_{2}}^{p}(\mu)$ coincide and their norms are equivalent.

Proof. It suffices to verify that

$$
\|f\|_{\mathrm{RBMO}_{\rho_{2}}^{p}(\mu)} \leqslant\|f\|_{\mathrm{RBMO}_{\rho_{1}}^{p}(\mu)} \lesssim\|f\|_{\mathrm{RBMO}_{\rho_{2}}^{p}(\mu)} .
$$

The first inequality is obvious, so we prove only the second one, which will be deduced from the argument used in the proof of $\left[\mathbf{9}\right.$, Lemma 2.6]. Let $f \in \operatorname{RBMO}_{\rho_{2}}^{p}(\mu)$. For each fixed cube $Q$ and $x \in Q \cap \operatorname{supp} \mu$, let $Q_{x}$ be a cube centred at $x$ with side length $l(Q)\left(\rho_{1}-1\right) /\left(10 \rho_{2}\right)$. Then $l\left(\rho_{2} Q_{x}\right)=l(Q)\left(\rho_{1}-1\right) / 10$ and so $\rho_{2} Q_{x} \subset \rho_{1} Q$. Applying the Besicovich covering lemma, we see that there exists a family of points $\left\{x_{j}\right\}_{j=1}^{N}$, $x_{j} \in(Q \cap \operatorname{supp} \mu)$, such that

(a) the $\left\{Q_{x_{j}}\right\}_{j=1}^{N}$ are almost disjoint;

(b) $(Q \cap \operatorname{supp} \mu) \subset \bigcup_{j} Q_{x_{j}}$.

Observe that $Q_{x_{j}}$ and $Q$ have comparable side length and

$$
\begin{aligned}
\left|f_{Q_{x_{j}}}-f_{Q}\right| & \leqslant\left|f_{Q_{x_{j}}}-f_{\rho_{1} Q}\right|+\left|f_{\rho_{1} Q}-f_{Q}\right| \\
& \lesssim\|f\|_{\operatorname{RBMO}_{\rho_{2}}^{p}(\mu)}\left(\delta_{Q_{x_{j}}, \rho_{1} Q}+\delta_{Q, \rho_{1} Q}\right) \\
& \lesssim\|f\|_{\operatorname{RBMO}_{\rho_{2}}^{p}(\mu)} .
\end{aligned}
$$


It then follows that

$$
\begin{aligned}
\int_{Q_{x_{j}}}\left|f(x)-f_{Q}\right|^{p} \mathrm{~d} \mu(x) & \leqslant \int_{Q_{x_{j}}}\left|f(x)-f_{Q_{x_{j}}}\right|^{p} \mathrm{~d} \mu(x)+\mu\left(Q_{x_{j}}\right)\left|f_{Q_{x_{j}}}-f_{Q}\right|^{p} \\
& \lesssim \mu\left(\rho_{2} Q_{x_{j}}\right)\|f\|_{\mathrm{RBMO}_{\rho_{2}}^{p}(\mu)}^{p} .
\end{aligned}
$$

Summing the last inequality over all $j$ then yields

$$
\begin{aligned}
\int_{Q}\left|f(x)-f_{Q}\right|^{p} \mathrm{~d} \mu(x) & \leqslant \sum_{j=1}^{N} \int_{Q_{x_{j}}}\left|f(x)-f_{Q}\right|^{p} \mathrm{~d} \mu(x) \\
& \lesssim \sum_{j=1}^{N} \mu\left(\rho_{2} Q_{x_{j}}\right)\|f\|_{\mathrm{RBMO}_{\rho_{2}}^{p}(\mu)}^{p} \\
& \lesssim N \mu\left(\rho_{1} Q\right)\|f\|_{\mathrm{RBMO}_{\rho_{2}}^{p}(\mu)}^{p},
\end{aligned}
$$

where, in the last inequality, we have invoked the fact that $\rho_{2} Q_{x_{j}} \subset \rho_{1} Q$. Since $N$ is a fixed positive integer depending only on $\rho_{1}, \rho_{2}$ and $d$ (see $[\mathbf{9}$, p. 99]), the desired result then follows from the last estimate directly.

Lemma 2.2. Let $\rho \in(1, \infty)$ and $p \in(0, \infty)$. Suppose that $f \in \operatorname{RBMO}_{\rho}^{p}(\mu)$ and let $\left\{f_{Q}\right\}$ be a collection of numbers satisfying (1.5) and (1.6). For each positive integer $m$, set

$$
f_{m}(x)= \begin{cases}f(x) & \text { if }|f(x)| \leqslant m \\ m \frac{f(x)}{|f(x)|} & \text { if }|f(x)|>m\end{cases}
$$

and

$$
f_{m, Q}= \begin{cases}f_{Q} & \text { if }\left|f_{Q}\right| \leqslant m \\ m \frac{f_{Q}}{\left|f_{Q}\right|} & \text { if }\left|f_{Q}\right|>m\end{cases}
$$

Then

$$
\sup _{Q} \frac{1}{\mu(\rho Q)} \int_{Q}\left|f_{m}(x)-f_{m, Q}\right|^{p} \mathrm{~d} \mu(x) \leqslant 3\|f\|_{\operatorname{RBMO}_{\rho}^{p}(\mu)}^{p},
$$

and, for any two cubes $Q \subset R$,

$$
\left|f_{m, Q}-f_{m, R}\right| \leqslant 3 \delta_{Q, R}\|f\|_{\mathrm{RBMO}_{\rho}^{p}(\mu)} .
$$

This lemma can be proved by a straightforward computation. We omit the details for brevity.

The following result is $[\mathbf{9}$, Lemma 2.7].

Lemma 2.3. Let $\rho \in(1, \infty)$. Then $\operatorname{RBMO}(\mu)$ and $\operatorname{RBMO}_{\rho}^{1}(\mu)$ coincide and their norms are equivalent. 
Proof of Theorem 1.4. Note that by the John-Nirenberg inequality established by Tolsa [9], for any $p \in(0, \infty)$ and $\rho \in(1, \infty), \operatorname{RBMO}(\mu) \subset \operatorname{RBMO}_{\rho}^{p}(\mu)$ and, by the Hölder inequality, for $p \in[1, \infty), \operatorname{RBMO}_{\rho}^{p}(\mu) \subset \operatorname{RBMO}(\mu)$. Thus, we need to prove only that, for each fixed $\rho \in(1, \infty), p \in(0,1)$ and any $f \in \operatorname{RBMO}_{\rho}^{p}(\mu)$,

$$
\|f\|_{\mathrm{RBMO}(\mu)} \lesssim\|f\|_{\mathrm{RBMO}_{\rho}^{p}(\mu)} .
$$

By Lemmas 2.1 and 2.3, it suffices to prove that if $f$ and a collection of numbers $\left\{f_{Q}\right\}$ satisfy the estimate (1.5) with $\rho=\frac{4}{3}$ and the estimate (1.6), then

$$
\sup _{Q} \frac{1}{\mu(2 Q)} \int_{Q}\left|f(x)-f_{Q}\right| \mathrm{d} \mu(x) \lesssim\|f\|_{\mathrm{RBMO}_{4 / 3}^{p}(\mu)} .
$$

For a fixed $f \in \operatorname{RBMO}_{4 / 3}^{p}(\mu)$ and a cube $Q$, let $B$ be a positive constant to be determined later. As in [9, p. 108], applying the Besicovich covering lemma, we have

$$
\left\{x \in Q:\left|f(x)-f_{Q}\right|>B\|f\|_{\mathrm{RBMO}_{4 / 3}^{p}(\mu)}\right\} \subset \bigcup_{j} Q_{j},
$$

where $\left\{Q_{j}\right\}$ is a sequence of cubes with almost disjoint interiors, $Q_{j} \subset \frac{3}{2} Q, l\left(Q_{j}\right) \leqslant$ $\frac{1}{10} l(Q)$, and $Q_{j}$ is a maximal doubling cube which satisfies

$$
\frac{1}{\mu\left(Q_{j}\right)} \int_{Q_{j}}\left|f(x)-f_{Q}\right|^{p} \mathrm{~d} \mu(x)>B^{p}\|f\|_{\mathrm{RBMO}_{4 / 3}^{p}(\mu)}^{p} .
$$

Therefore,

$$
\begin{aligned}
\sum_{j} \mu\left(Q_{j}\right) & \leqslant \frac{1}{B^{p}\|f\|_{\mathrm{RBMO}_{4 / 3}^{p}(\mu)}^{p}} \sum_{j} \int_{Q_{j}}\left|f(x)-f_{Q}\right|^{p} \mathrm{~d} \mu(x) \\
& \leqslant \frac{1}{B^{p}\|f\|_{\mathrm{RBMO}_{4 / 3}^{p}(\mu)}^{p}} \int_{3 Q / 2}\left|f(x)-f_{Q}\right|^{p} \mathrm{~d} \mu(x) \\
& \leqslant \frac{1}{B^{p}\|f\|_{\mathrm{RBMO}_{4 / 3}^{p}(\mu)}^{p}} \int_{3 Q / 2}\left|f(x)-f_{3 Q / 2}\right|^{p} \mathrm{~d} \mu(x) \\
& \leqslant \frac{\mu(3 Q / 2)}{B^{p}\|f\|_{\mathrm{RBMO}_{4 / 3}^{p}(\mu)}^{p}}\left|f_{Q}-f_{3 Q / 2}\right|^{p} \\
& \leqslant \frac{C_{3}}{B^{p}} \mu(2 Q) .
\end{aligned}
$$

We now choose $B$ large enough such that $C_{3} 2^{d+1} / B^{p} \leqslant \frac{1}{2}$. It follows that

$$
\sum_{j} \mu\left(2 Q_{j}\right) \leqslant \frac{1}{2} \mu(2 Q) .
$$

Now we claim that, for each fixed $j$,

$$
\left|f_{Q}-f_{Q_{j}}\right| \lesssim\|f\|_{\mathrm{RBMO}_{4 / 3}^{p}(\mu)} .
$$

To prove this, we consider the following three cases. 
Case $1\left(l\left(\widetilde{2 Q_{j}}\right) \leqslant 1^{-1} l(Q)\right)$. From the choice of $Q_{j}$, it follows that

$$
\frac{1}{\mu\left(\widetilde{2 Q_{j}}\right)} \int_{\widetilde{2 Q_{j}}}\left|f(x)-f_{Q}\right|^{p} \mathrm{~d} \mu(x) \leqslant B^{p}\|f\|_{\mathrm{RBMO}_{4 / 3}^{p}(\mu)}^{p} .
$$

Observing that

$$
\begin{aligned}
\left|f_{Q_{j}}-f_{\overparen{2 Q_{j}}}\right| & \leqslant\left|f_{Q_{j}}-f_{2 Q_{j}}\right|+\left|f_{2 Q_{j}}-f_{\widetilde{2 Q_{j}}}\right| \\
& \lesssim \delta_{Q_{j}, 2 Q_{j}}\|f\|_{\mathrm{RBMO}_{4 / 3}^{p}(\mu)}+\delta_{2 Q_{j}, \widetilde{2 Q_{j}}}\|f\|_{\mathrm{RBMO}_{4 / 3}^{p}(\mu)},
\end{aligned}
$$

we then have

$$
\left|f_{Q_{j}}-f_{\widetilde{2 Q_{j}}}\right| \lesssim\|f\|_{\mathrm{RBMO}_{4 / 3}^{p}(\mu)}
$$

Thus,

$$
\begin{aligned}
\left|f_{Q}-f_{Q_{j}}\right|^{p} & \leqslant \frac{1}{\mu\left(\widetilde{2 Q_{j}}\right)} \int_{\widetilde{2 Q_{j}}}\left|f(x)-f_{Q}\right|^{p} \mathrm{~d} \mu(x)+\frac{1}{\mu\left(\widetilde{2 Q_{j}}\right)} \int_{\widetilde{2 Q_{j}}}\left|f(x)-f_{Q_{j}}\right|^{p} \mathrm{~d} \mu(x) \\
& \leqslant B^{p}\|f\|_{\mathrm{RBMO}_{4 / 3}^{p}(\mu)}^{p}+\left|f_{Q_{j}}-f_{\widetilde{2 Q_{j}}}\right|^{p}+\frac{1}{\mu\left(\widetilde{2 Q_{j}}\right)} \int_{\widetilde{2 Q_{j}}}\left|f(x)-f_{\widetilde{2 Q_{j}}}\right|^{p} \mathrm{~d} \mu(x) \\
& \lesssim B^{p}\|f\|_{\mathrm{RBMO}_{4 / 3}^{p}(\mu)}^{p} .
\end{aligned}
$$

Case $2\left(\mathbf{1 0}^{-1} \boldsymbol{l}(Q)<\boldsymbol{l}\left(\widetilde{\mathbf{2 Q _ { j }}}\right) \leqslant \mathbf{1 0 l}(Q)\right)$. By $(2.5)$ and the fact that $\widetilde{2 Q_{j}}$ and $Q$ have comparable side length, we know that

$$
\left|f_{Q}-f_{Q_{j}}\right| \leqslant\left|f_{Q_{j}}-f_{\widetilde{2 Q_{j}}}\right|+\left|f_{\widetilde{2 Q_{j}}}-f_{Q}\right| \lesssim\|f\|_{\mathrm{RBMO}_{4 / 3}^{p}(\mu)} .
$$

Case $3\left(\boldsymbol{l}\left(\widetilde{2 Q_{j}}\right)>\mathbf{1 0 l}(Q)\right)$. Let $m$ be a positive integer such that $Q \subset 2^{m} Q_{j}$ and $l(Q) \sim l\left(2^{m} Q_{j}\right) \leqslant l\left(\widetilde{2 Q_{j}}\right)$. Write

$$
\left|f_{Q}-f_{Q_{j}}\right| \leqslant\left|f_{Q_{j}}-f_{2 Q_{j}}\right|+\left|f_{2 Q_{j}}-f_{2^{m} Q_{j}}\right|+\left|f_{Q}-f_{2^{m} Q_{j}}\right| .
$$

Note that $2^{m} Q_{j} \subset \widetilde{2 Q_{j}}$. It follows that

$$
\left|f_{2 Q_{j}}-f_{2^{m} Q_{j}}\right| \leqslant \delta_{2 Q_{j}, 2^{m} Q_{j}}\|f\|_{\mathrm{RBMO}_{4 / 3}^{p}(\mu)} \leqslant \delta_{2 Q_{j}, \widetilde{2 Q_{j}}}\|f\|_{\mathrm{RBMO}_{4 / 3}^{p}(\mu)} \lesssim\|f\|_{\mathrm{RBMO}_{4 / 3}^{p}(\mu)} .
$$

On the other hand, the fact that $Q \subset 2^{m} Q_{j}$ and $l(Q) \sim l\left(2^{m} Q_{j}\right)$ tells us that $\delta_{Q, 2^{m}} Q_{j} \lesssim 1$ and that

$$
\left|f_{Q}-f_{2^{m} Q_{j}}\right| \leqslant\|f\|_{\mathrm{RBMO}_{4 / 3}^{p}(\mu)} \delta_{Q, 2^{m} Q_{j}} \lesssim\|f\|_{\mathrm{RBMO}_{4 / 3}^{p}(\mu)},
$$

which gives the desired estimate (2.4) in this case.

We can now conclude the proof of Theorem 1.4. Set

$$
D=\sup _{I} \frac{1}{\mu(2 I)} \int_{I}\left|f(x)-f_{I}\right| \mathrm{d} \mu(x)
$$


where the supremum is taken over all cubes $I \subset \mathbb{R}^{d}$. The estimates (2.3) and (2.4) state that

$$
\begin{aligned}
& \frac{1}{\mu(2 Q)} \int_{Q}\left|f(x)-f_{Q}\right| \mathrm{d} \mu(x) \\
& \quad \leqslant \frac{1}{\mu(2 Q)} \int_{Q \backslash \cup_{j} Q_{j}}\left|f(x)-f_{Q}\right| \mathrm{d} \mu(x)+\frac{1}{\mu(2 Q)} \sum_{j} \int_{Q_{j}}\left|f(x)-f_{Q}\right| \mathrm{d} \mu(x) \\
& \quad \leqslant B\|f\|_{\mathrm{RBMO}_{4 / 3}^{p}(\mu)}+\frac{1}{\mu(2 Q)} \sum_{j} \mu\left(Q_{j}\right)\left|f_{Q}-f_{Q_{j}}\right|+\frac{1}{\mu(2 Q)} \sum_{j} \int_{Q_{j}}\left|f(x)-f_{Q_{j}}\right| \mathrm{d} \mu(x) \\
& \quad \leqslant C\|f\|_{\mathrm{RBMO}_{4 / 3}^{p}(\mu)}+\frac{D}{\mu(2 Q)} \sum_{j} \mu\left(2 Q_{j}\right)
\end{aligned}
$$

where $C>0$ is independent of $Q$ and $f$. This in turn implies that, for all $f \in L^{\infty}(\mu)$,

$$
D \lesssim\|f\|_{\mathrm{RBMO}_{4 / 3}^{p}(\mu)}
$$

For a general $f \in \mathrm{RBMO}_{4 / 3}^{p}(\mu)$, letting $f_{m}$ and $f_{m, Q}$ be as in Lemma 2.2, we then have $f_{m} \in L^{\infty}(\mu)$ and so, for any cube $Q$,

$$
\frac{1}{\mu(2 Q)} \int_{Q}\left|f_{m}(x)-f_{m, Q}\right| \mathrm{d} \mu(x) \lesssim\left\|f_{m}\right\|_{\mathrm{RBMO}_{4 / 3}^{p}(\mu)} \lesssim\|f\|_{\mathrm{RBMO}_{4 / 3}^{p}(\mu)} .
$$

Since

$$
\lim _{m \rightarrow \infty} \frac{1}{\mu(2 Q)} \int_{Q}\left|f_{m}(x)-f_{m, Q}\right| \mathrm{d} \mu(x)=\frac{1}{\mu(2 Q)} \int_{Q}\left|f(x)-f_{Q}\right| \mathrm{d} \mu(x),
$$

we see that the inequality (2.1) holds for any $f \in \operatorname{RBMO}_{4 / 3}^{p}(\mu)$, which completes the proof of Theorem 1.4.

\section{Proof of Theorem 1.9}

To prove Theorem 1.9, we need the following lemma.

Lemma 3.1. Let $K$ satisfy (1.7) and (1.8), let $T_{\varepsilon}$ be defined by (1.10) and let $\sigma \in(0,1)$. If Theorem 1.9 (iii) with $\rho=\frac{3}{2}$ is true, then, for any $\varepsilon>0$ and any bounded function $f$ with compact support, $\left|T_{\varepsilon} f\right|^{\sigma} \in \operatorname{RBMO}(\mu)$ and

$$
\left\|\left|T_{\varepsilon} f\right|^{\sigma}\right\|_{\mathrm{RBMO}(\mu)} \leqslant C\|f\|_{L^{\infty}(\mu)}^{\sigma}
$$

with $C>0$ independent of $f$ and $\varepsilon$.

Proof. By homogeneity, we may assume that $\|f\|_{L^{\infty}(\mu)}=1$. Set

$$
h_{Q, \sigma}=m_{Q}\left(\left|T_{\varepsilon}\left(f \chi_{\mathbb{R}^{d} \backslash Q / 3}\right)\right|^{\sigma}\right) .
$$

By Lemma 2.3, we see that the proof of (3.1) can be reduced to proving that

$$
\left.\frac{1}{\mu(2 Q)} \int_{Q}|| T_{\varepsilon} f(x)\right|^{\sigma}-h_{Q, \sigma} \mid \mathrm{d} \mu(x) \lesssim 1
$$


and, for any two cubes $Q$ and $R$ with $Q \subset R$,

$$
\left|h_{Q, \sigma}-h_{R, \sigma}\right| \lesssim \delta_{Q, R}
$$

We first prove (3.2). For a fixed cube $Q$ and bounded function $f$ with compact support and $\|f\|_{L^{\infty}(\mu)}=1$, decompose $f$ as

$$
f(x)=f(x) \chi_{4 Q / 3}(x)+f(x) \chi_{\mathbb{R}^{d} \backslash 4 Q / 3}(x)=f_{1}(x)+f_{2}(x) .
$$

Write

$$
\begin{aligned}
& \left.\frac{1}{\mu(2 Q)} \int_{Q}|| T_{\varepsilon} f(x)\right|^{\sigma}-h_{Q, \sigma} \mid \mathrm{d} \mu(x) \\
& \quad \leqslant\left.\frac{1}{\mu(2 Q)} \int_{Q}|| T_{\varepsilon} f(x)\right|^{\sigma}-\left|T_{\varepsilon} f_{2}(x)\right|^{\sigma}\left|\mathrm{d} \mu(x)+\frac{1}{\mu(2 Q)} \int_{Q}\right|\left|T_{\varepsilon} f_{2}(x)\right|^{\sigma}-h_{Q, \sigma} \mid \mathrm{d} \mu(x) \\
& \quad \leqslant \frac{1}{\mu(2 Q)} \int_{Q}\left|T_{\varepsilon} f_{1}(x)\right|^{\sigma} \mathrm{d} \mu(x)+\left.\frac{1}{\mu(2 Q)} \int_{Q}|| T_{\varepsilon} f_{2}(x)\right|^{\sigma}-h_{Q, \sigma} \mid \mathrm{d} \mu(x) .
\end{aligned}
$$

Our hypothesis now states that

$$
\frac{1}{\mu(2 Q)} \int_{Q}\left|T_{\varepsilon} f_{1}(x)\right|^{\sigma} \mathrm{d} \mu(x) \leqslant \frac{1}{\mu(2 Q)} \int_{4 Q / 3}\left|T_{\varepsilon} f_{1}(x)\right|^{\sigma} \mathrm{d} \mu(x) \lesssim 1 .
$$

On the other hand, for any $x, y \in Q$, a trivial computation gives

$$
\begin{aligned}
\left.|| T_{\varepsilon} f_{2}(x)\right|^{\sigma}-\left|T_{\varepsilon} f_{2}(y)\right|^{\sigma} \mid & \leqslant\left|T_{\varepsilon} f_{2}(x)-T_{\varepsilon} f_{2}(y)\right|^{\sigma} \\
& \leqslant\left\{\int_{\mathbb{R}^{d} \backslash 4 Q / 3}|K(x, z)-K(y, z)| \mathrm{d} \mu(z)\right\}^{\sigma} \\
& \lesssim 1 .
\end{aligned}
$$

Thus, for any $x \in Q$,

$$
\left.|| T_{\varepsilon} f_{2}(x)\right|^{\sigma}-h_{Q, \sigma}\left|=\frac{1}{\mu(Q)}\right| \int_{Q}\left(\left|T_{\varepsilon} f_{2}(y)\right|^{\sigma}-\left|T_{\varepsilon} f_{2}(x)\right|^{\sigma}\right) \mathrm{d} \mu(y) \mid \lesssim 1,
$$

which in turn leads to

$$
\left.\frac{1}{\mu(2 Q)} \int_{Q}|| T_{\varepsilon} f_{2}(x)\right|^{\sigma}-h_{Q, \sigma} \mid \mathrm{d} \mu(x) \lesssim 1
$$

Inequality (3.2) then holds.

Now we turn our attention to the estimate (3.3). For two cubes $Q$ and $R$ with $Q \subset R$, we denote $N_{Q, R}+1$ simply by $N$. Write

$$
\begin{aligned}
& \left|h_{Q, \sigma}-h_{R, \sigma}\right| \\
& \leqslant\left|m_{Q}\left(\left|T_{\varepsilon}\left(f \chi_{\mathbb{R}^{d} \backslash 4 Q / 3}\right)\right|^{\sigma}\right)-m_{Q}\left(\left|T_{\varepsilon}\left(f \chi_{\mathbb{R}^{d} \backslash 2^{N} Q}\right)\right|^{\sigma}\right)\right| \\
& \quad+\left|m_{Q}\left(\left|T_{\varepsilon}\left(f \chi_{\mathbb{R}^{d} \backslash 2^{N} Q}\right)\right|^{\sigma}\right)-m_{R}\left(\left|T_{\varepsilon}\left(f \chi_{\mathbb{R}^{d} \backslash 2^{N} Q}\right)\right|^{\sigma}\right)\right| \\
& \quad+\left|m_{R}\left(\left|T_{\varepsilon}\left(f \chi_{\mathbb{R}^{d} \backslash 4 R / 3}\right)\right|^{\sigma}\right)-m_{R}\left(\left|T_{\varepsilon}\left(f \chi_{\mathbb{R}^{d} \backslash 2^{N} Q}\right)\right|^{\sigma}\right)\right|
\end{aligned}
$$




$$
\begin{aligned}
\leqslant & m_{Q}\left(\left|T_{\varepsilon}\left(f \chi_{2^{N} Q \backslash 4 Q / 3}\right)\right|^{\sigma}\right)+\left|m_{Q}\left(\left|T_{\varepsilon}\left(f \chi_{\mathbb{R}^{d} \backslash 2^{N} Q}\right)\right|^{\sigma}\right)-m_{R}\left(\left|T_{\varepsilon}\left(f \chi_{\mathbb{R}^{d} \backslash 2^{N} Q}\right)\right|^{\sigma}\right)\right| \\
& \quad+m_{R}\left(\left|T_{\varepsilon}\left(f \chi_{2^{N} Q \backslash 4 R / 3}\right)\right|^{\sigma}\right) \\
=I+ & J+H .
\end{aligned}
$$

The estimate for $H$ is easy. In fact, by (1.1), we see that, for each fixed $y \in R$,

$$
\left|T_{\varepsilon}\left(f \chi_{2^{N} Q \backslash 4 R / 3}\right)(y)\right| \lesssim \frac{\mu\left(2^{N} Q\right)}{[l(R)]^{n}} \lesssim 1,
$$

which implies that $H \lesssim 1$.

Recall that $N_{Q, R}$ is the least integer $k$ such that $2^{k} Q \supset R$. A trivial computation involving the regularity condition (1.8) proves that, for any $x \in Q$ and $y \in R$,

$$
\begin{aligned}
\|\left. T_{\varepsilon}\left(f \chi_{\mathbb{R}^{d} \backslash 2^{N} Q}\right)(x)\right|^{\sigma}-\left|T_{\varepsilon}\left(f \chi_{\mathbb{R}^{d} \backslash 2^{N} Q}\right)(y)\right|^{\sigma} \mid & \leqslant\left|T_{\varepsilon}\left(f \chi_{\mathbb{R}^{d} \backslash 2^{N} Q}\right)(x)-T_{\varepsilon}\left(f \chi_{\mathbb{R}^{d} \backslash 2^{N} Q}\right)(y)\right|^{\sigma} \\
& \leqslant\left\{\int_{\mathbb{R}^{d} \backslash 2^{N} Q}|K(x, z)-K(y, z)| \mathrm{d} \mu(z)\right\}^{\sigma} \\
& \lesssim 1 .
\end{aligned}
$$

Using this result, and the fact that

$$
\begin{aligned}
& \left|m_{Q}\left(\left|T_{\varepsilon}\left(f \chi_{\mathbb{R}^{d} \backslash 2^{N} Q}\right)\right|^{\sigma}\right)-m_{R}\left(\left|T_{\varepsilon}\left(f \chi_{\mathbb{R}^{d} \backslash 2^{N} Q}\right)\right|^{\sigma}\right)\right| \\
& \quad \leqslant\left.\frac{1}{\mu(Q) \mu(R)} \int_{Q} \int_{R}|| T_{\varepsilon}\left(f \chi_{\mathbb{R}^{d} \backslash 2^{N} Q}\right)(x)\right|^{\sigma}-\left|T_{\varepsilon}\left(f \chi_{\mathbb{R}^{d} \backslash 2^{N} Q}\right)(y)\right|^{\sigma} \mid \mathrm{d} \mu(x) \mathrm{d} \mu(y),
\end{aligned}
$$

gives the desired estimate for $J$, namely, $J \lesssim 1$.

Finally, we can verify that, for any $x \in Q$,

$$
\begin{aligned}
\left|T_{\varepsilon}\left(f \chi_{2^{N} Q \backslash 4 Q / 3}\right)(x)\right| \lesssim & \int_{2^{N} Q \backslash 2^{N-1} Q} \frac{1}{|x-z|^{n}} \mathrm{~d} \mu(z) \\
& +\int_{2^{N-1} Q \backslash 2 Q} \frac{1}{|x-z|^{n}} \mathrm{~d} \mu(z)+\int_{2 Q \backslash 4 Q / 3} \frac{1}{|x-z|^{n}} \mathrm{~d} \mu(z) \\
\lesssim & \frac{\mu\left(2^{N} Q\right)}{\left[l\left(2^{N-1} Q\right)\right]^{n}}+\sum_{k=1}^{N-1} \frac{\mu\left(2^{k} Q\right)}{\left[l\left(2^{k-1} Q\right)\right]^{n}}+\frac{\mu(2 Q)}{[l(4 Q / 3)]^{n}} \\
\lesssim & \delta_{Q, R},
\end{aligned}
$$

and so

$$
I \lesssim \delta_{Q, R}^{\sigma} \lesssim \delta_{Q, R}
$$

since $\sigma \in(0,1)$ and $\delta_{Q, R} \geqslant 1$. This finishes the proof of Lemma 3.1.

Proof of Theorem 1.9. By [9, Theorem 8.1 and Theorem 7.2], we know that (iv) $\Longrightarrow$ (v) and (v) $\Longrightarrow$ (i). Thus, we need only prove that (i) $\Longrightarrow$ (ii) $\Longrightarrow$ (iii) $\Longrightarrow$ (iv). 
(i) $\Longrightarrow$ (ii). We will prove that if (i) is true, then (ii) holds for $r=1$. Without loss of generality, we may assume that $\rho=2$ in (ii). Let $Q \subset \mathbb{R}^{d}$ be a cube and let $a$ be a bounded function supported on $Q$. We employ the idea used in [9, p. 137] and consider the following two cases.

Case $1(l(Q) \leqslant \operatorname{diam}(\operatorname{supp} \boldsymbol{\mu}) / \mathbf{2 0})$. Let $x_{0} \in \operatorname{supp} \mu$ be a point in $\mathbb{R}^{d} \backslash(5 Q)^{\circ}$ which is closest to $Q$, where $(5 Q)^{\circ}$ is the interior of $5 Q$. Set $d_{0}=\operatorname{dist}\left(x_{0}, Q\right)$. We assume that $x_{0}$ is a point such that some cube with side length $2^{-k} d_{0}, k \geqslant 2$, and centred at $x_{0}$ is doubling. Otherwise, we take $y_{0} \in \operatorname{supp} \mu \cap B\left(x_{0}, l(Q) / 100\right)$, which satisfies this condition, and then interchange $x_{0}$ and $y_{0}$. Denote by $R$ the cube concentric with $Q$ with side length $\max \left\{10 d_{0}, l(\tilde{Q})\right\}$. It is easy to verify that $\delta_{Q, R} \lesssim 1$. Let $Q_{0}$ be the biggest doubling cube centred at $x_{0}$ with side length $2^{-k} d_{0}, k \geqslant 2$. Then $Q_{0} \subset R, \delta_{Q_{0}, R} \lesssim 1$ and $\operatorname{dist}\left(Q_{0}, Q\right) \geqslant l(Q)$. Set

$$
b(y)=a(y)+C_{Q_{0}} \chi_{Q_{0}}(y)
$$

with $C_{Q_{0}}=-\left[\mu\left(Q_{0}\right)\right]^{-1} \int_{\mathbb{R}^{d}} a(y) \mathrm{d} \mu(y)$. It is easy to verify that, for $x \in Q$,

$$
\left|T_{\varepsilon}\left(C_{Q_{0}} \chi_{Q_{0}}\right)(x)\right| \lesssim\left|C_{Q_{0}}\right| \frac{\mu\left(Q_{0}\right)}{\left[\operatorname{dist}\left(x, Q_{0}\right)\right]^{n}} \leqslant C_{4}\|a\|_{L^{\infty}(\mu)} .
$$

If $\lambda \leqslant 2 C_{4}\|a\|_{L^{\infty}(\mu)}$, it is obvious that

$$
\mu\left(\left\{x \in Q:\left|T_{\varepsilon} a(x)\right|>\lambda\right\}\right) \lesssim \mu(Q) \lambda^{-1}\|a\|_{L^{\infty}(\mu)} .
$$

Thus, we may assume that $\lambda>2 C_{4}\|a\|_{L^{\infty}(\mu)}$. Observe that $b$ is an $H^{1}(\mu)$-atomic block and

$$
|b|_{H_{\mathrm{atb}}^{1, \infty}(\mu)} \leqslant \delta_{Q, R}\|a\|_{L^{\infty}(\mu)} \mu(2 Q)+\delta_{Q_{0}, R}\left|C_{Q_{0}}\right| \mu\left(2 Q_{0}\right) \lesssim\|a\|_{L^{\infty}(\mu)} \mu(2 Q)+\|a\|_{L^{1}(\mu)},
$$

since $Q_{0}$ is doubling and $\left|C_{Q_{0}}\right| \mu\left(Q_{0}\right)=\|a\|_{L^{1}(\mu)}$. It follows that

$$
\begin{aligned}
\mu\left(\left\{x \in Q:\left|T_{\varepsilon} a(x)\right|>\lambda\right\}\right) & \leqslant \mu\left(\left\{x \in Q:\left|T_{\varepsilon} b(x)\right|>\lambda / 2\right\}\right) \\
& \lesssim \lambda^{-1}\|b\|_{H_{\text {atb }}^{1, \infty}(\mu)} \\
& \lesssim \lambda^{-1}\|a\|_{L^{\infty}(\mu)} \mu(2 Q) .
\end{aligned}
$$

Case $2(l(Q)>\operatorname{diam}(\operatorname{supp} \boldsymbol{\mu}) / \mathbf{2 0})$. Since $Q$ is centred at some point of $\operatorname{supp} \mu$, we may assume that $l(Q) \leqslant 4 \operatorname{diam}(\operatorname{supp} \mu)$. Then $Q \cap \operatorname{supp} \mu$ can be covered by a finite number of cubes $\left\{Q_{j}\right\}_{j}$, centred at some points in $\operatorname{supp} \mu$ with side length $l(Q) / 200$ and satisfies $4 Q_{j} \subset 2 Q$. The number of such cubes $\left\{Q_{j}\right\}_{j}$ is less than a fixed positive integer $N$ depending only on $d$. Set

$$
a_{j}(x)=\frac{\chi_{Q_{j}}(x)}{\sum_{k} \chi_{Q_{k}}(x)} a(x)
$$


and write

$$
\begin{aligned}
\mu\left(\left\{x \in Q:\left|T_{\varepsilon} a(x)\right|>\lambda\right\}\right) \leqslant & \sum_{j=1}^{N} \mu\left(\left\{x \in Q:\left|T_{\varepsilon} a_{j}(x)\right|>\lambda / N\right\}\right) \\
\leqslant & \sum_{j=1}^{N} \mu\left(\left\{x \in Q \backslash 2 Q_{j}:\left|T_{\varepsilon} a_{j}(x)\right|>\lambda / N\right\}\right) \\
& +\sum_{j=1}^{N} \mu\left(\left\{x \in 2 Q_{j}:\left|T_{\varepsilon} a_{j}(x)\right|>\lambda / N\right\}\right) .
\end{aligned}
$$

Note that, for any $x \notin 2 Q_{j}$,

$$
\left|T_{\varepsilon} a_{j}(x)\right| \lesssim\|a\|_{L^{\infty}(\mu)} \mu\left(Q_{j}\right)\left[l\left(Q_{j}\right)\right]^{-n} \lesssim\|a\|_{L^{\infty}(\mu)} .
$$

This, together with (1.1), leads to

$$
\begin{aligned}
\mu\left(\left\{x \in Q \backslash 2 Q_{j}:\left|T_{\varepsilon} a_{j}(x)\right|>\lambda / N\right\}\right) & \leqslant N \lambda^{-1} \int_{Q \backslash 2 Q_{j}}\left|T_{\varepsilon} a_{j}(x)\right| \mathrm{d} \mu(x) \\
& \lesssim N \lambda^{-1} \mu(Q)\|a\|_{L^{\infty}(\mu)} .
\end{aligned}
$$

On the other hand, by the conclusion in Case 1, we have

$$
\mu\left(\left\{x \in 2 Q_{j}:\left|T_{\varepsilon} a_{j}(x)\right|>\lambda / N\right\}\right) \lesssim \lambda^{-1}\|a\|_{L^{\infty}(\mu)} \mu\left(4 Q_{j}\right) .
$$

Finally, in this case we obtain

$$
\mu\left(\left\{x \in Q:\left|T_{\varepsilon} a(x)\right|>\lambda\right\}\right) \lesssim \lambda^{-1} \mu(2 Q)\|a\|_{L^{\infty}(\mu)} .
$$

(ii) $\Longrightarrow$ (iii). Assume that (ii) is true. Choose $\sigma \in(0, \min \{1, \nu\})$. If $f$ is a bounded function with support contained in a cube $Q$, a trivial computation gives that

$$
\begin{aligned}
\int_{Q}\left|T_{\varepsilon} f(x)\right|^{\sigma} \mathrm{d} \mu(x) & =\sigma \int_{0}^{\|f\|_{L^{\infty}(\mu)}} \mu\left(\left\{x \in Q:\left|T_{\varepsilon} f(x)\right|>\lambda\right\}\right) \lambda^{\sigma-1} \mathrm{~d} \lambda \\
& +\sigma \int_{\|f\|_{L^{\infty}(\mu)}}^{\infty} \mu\left(\left\{x \in Q:\left|T_{\varepsilon} f(x)\right|>\lambda\right\}\right) \lambda^{\sigma-1} \mathrm{~d} \lambda \\
& \lesssim \mu(Q)\|f\|_{L^{\infty}(\mu)}^{\sigma}+\mu(\rho Q)\|f\|_{L^{\infty}(\mu)}^{\nu} \int_{\|f\|_{L^{\infty}(\mu)}}^{\infty} \lambda^{\sigma-\nu-1} \mathrm{~d} \lambda \\
& \lesssim \mu(\rho Q)\|f\|_{L^{\infty}(\mu)}^{\sigma} .
\end{aligned}
$$

(iii) $\Longrightarrow$ (iv). For simplicity, assume that (iii) is true with $\rho=\frac{3}{2}$. Now our goal is to prove that, for any $\varepsilon>0$ and bounded function $f$ with compact support,

$$
\left\|T_{\varepsilon} f\right\|_{\mathrm{RBMO}(\mu)} \lesssim\|f\|_{L^{\infty}(\mu)} .
$$


For each fixed $\varepsilon>0$ and bounded function $f$ with compact support, Lemma 3.1 tells us that $\left|T_{\varepsilon} f\right|^{\sigma} \in \operatorname{RBMO}(\mu)$, where $\sigma \in(0,1)$. By the John-Nirenberg inequality, we know that $T_{\varepsilon} f$ is locally $\mu$-integrable. For each fixed cube $Q \subset \mathbb{R}^{d}$, set

$$
h_{Q}=m_{Q}\left(T_{\varepsilon}\left(f \chi_{\mathbb{R}^{d} \backslash 4 Q / 3}\right)\right) .
$$

Repeating the argument used in the proof of Lemma 3.1, we can prove that, for any two cubes $Q$ and $R$ with $Q \subset R$,

$$
\left|h_{Q}-h_{R}\right| \lesssim \delta_{Q, R}\|f\|_{L^{\infty}(\mu)}
$$

On the other hand, as in the proof of Lemma 3.1, a straightforward computation proves that, for any cube $Q$,

$$
\frac{1}{\mu(2 Q)} \int_{Q}\left|T_{\varepsilon} f(x)-h_{Q}\right|^{\sigma} \mathrm{d} \mu(x) \lesssim\|f\|_{L^{\infty}(\mu)}^{\sigma} .
$$

This leads, via Theorem 1.4, to

$$
\left\|T_{\varepsilon} f\right\|_{\mathrm{RBMO}(\mu)} \lesssim\|f\|_{L^{\infty}(\mu)}
$$

which completes the proof of Theorem 1.9.

Proof of Corollary 1.10. By Theorem 1.9, it suffices to prove that condition (iii) in Theorem 1.9 is true. Let $Q$ be a fixed cube and $f$ be a bounded function with $\|f\|_{L^{\infty}(\mu)}=1$ and support contained in some cube $Q$. A trivial computation proves that, for any $\sigma \in(0,1)$,

$$
\begin{aligned}
\int_{Q}\left|T_{\varepsilon} f(x)\right|^{\sigma} \mathrm{d} \mu(x) & =\sigma \int_{0}^{1} \lambda^{\sigma-1} \mu\left(\left\{x \in Q:\left|T_{\varepsilon} f(x)\right|>\lambda\right\}\right) \mathrm{d} \lambda \\
& \quad+\sigma \int_{1}^{\infty} \lambda^{\sigma-1} \mu\left(\left\{x \in Q:\left|T_{\varepsilon} f(x)\right|>\lambda\right\}\right) \mathrm{d} \lambda \\
& \lesssim \mu(Q)+\left\{\int_{1}^{\infty} \lambda^{\sigma-1} \Phi(1 / \lambda) \mathrm{d} \lambda\right\} \int_{Q} \Phi(|f(x)|) \mathrm{d} \mu(x) \\
& \lesssim \mu(Q),
\end{aligned}
$$

and the desired estimate holds.

We end this section with the following remark.

Remark 3.2. By the same argument as above, it is easy to check that the conclusion of Theorem 1.9 is still true if we replace (1.8) by the following Hörmander condition: for all $y, y^{\prime} \in \mathbb{R}^{d}$,

$$
\int_{|x-y| \geqslant 2\left|y-y^{\prime}\right|}\left[\left|K(x, y)-K\left(x, y^{\prime}\right)\right|+\left|K(y, x)-K\left(y^{\prime}, x\right)\right|\right] \mathrm{d} \mu(x) \lesssim 1 .
$$

We omit the details for brevity.

Acknowledgements. D.Y. was supported by the National Science Foundation for Distinguished Young Scholars (no. 10425106) and NCET of the Ministry of Education (no. NCET-04-0142) of China. The authors thank the referee and the editor for many valuable remarks which made this article more readable. 


\section{References}

1. G. HU AND S. LiAng, Another characterization of the Hardy spaces with non-doubling measures, Math. Nachr. 279 (2006), 1797-1807.

2. G. Hu, Y. Meng AND D. YANG, New atomic characterization of $H^{1}$ space with nondoubling measures and its applications, Math. Proc. Camb. Phil. Soc. 138 (2005), 151171.

3. R. Long And L. YAng, BMO functions in spaces of homogeneous type, Sci. China A 27 (1984), 695-708.

4. J. Mateu, P. Mattila, A. Nicolau and J. Orobitg, BMO for nondoubling measures, Duke Math. J. 102 (2000), 533-565.

5. F. Nazarov, S. Treil and A. Volberg, Weak type estimates and Cotlar inequalities for Calderón-Zygmund operators on nonhomogeneous spaces, Int. Math. Res. Not. 9 (1998), 463-487.

6. F. Nazarov, S. Treil and A. Volberg, The $T b$-theorem on non-homogeneous spaces, Acta Math. 190 (2003), 151-239.

7. J. Orobitg And C. PÉREz, $A_{p}$ weights for nondoubling measures in $\mathbb{R}^{n}$ and applications, Trans. Am. Math. Soc. 354 (2002), 2013-2033.

8. J.-O. StrömberG, Bounded mean oscillation with Orlicz norms and duality of Hardy spaces, Indiana Univ. Math. J. 28 (1979), 511-544.

9. X. Tolsa, BMO, $H^{1}$ and Calderón-Zygmund operators for non doubling measures, Math. Ann. 319 (2001), 89-149.

10. X. TolsA, A proof of the weak $(1,1)$ inequality for singular integrals with non doubling measures based on a Calderón-Zygmund decomposition, Publ. Mat. 45 (2001), 163-174.

11. X. TOLSA, The space $H^{1}$ for non doubling measures in terms of a grand maximal operator, Trans. Am. Math. Soc. 355 (2003), 315-348.

12. X. TolsA, Painlevé's problem and semiadditivity of analytic capacity, Acta Math. 190 (2003), 105-149.

13. J. Verdera, The fall of the doubling condition in Calderón-Zygmund theory, Publ. Mat. (Extra) (2002), 275-292. 